\title{
Quality of Health Care Assessment
}

National Cancer Institute

\section{Source}

National Cancer Institute. Quality of Health Care Assessment. NCI Thesaurus. Code C41392.

The systemic measurement of the quality of health care that includes a broad set of perfomance measures such as the quality of services and the outcomes analysis for particular diseases and conditions, mortality and morbidity, patient safety, data on maternal and child health, as well as evaluation of quality of diagnosis, care excessability, implementation of proven evidence-based practices, quality of reporting systems for errors and adverse events, reliability and excessability of health and health care-related information, and health care organizations infrastructure. 